\title{
Musculoskeletal Examination Skills: Are We Still Interested?
}

\author{
Hani Almoallim ${ }^{1,2,3^{*}}$, Abdulhadi Gelidan ${ }^{1}$ \\ ${ }^{1}$ Department of Medicine, Umm Alqura University, Makkah, KSA; ${ }^{2}$ Department of Medicine, King Faisal Specialist Hospital, Jed- \\ dah, KSA; ${ }^{3}$ Alzaidi Chair of Research in Rheumatic Diseases, Umm Alqura University, Makkah, KSA. \\ Email: *Hanialmoallim@hotmail.com
}

Received May 25 ${ }^{\text {th }}$, 2012; revised June 26 ${ }^{\text {th }}$, 2012; accepted July $14^{\text {th }}, 2012$

\begin{abstract}
This article explores problems and solutions of Musculoskeletal (MSK) examinations skills and points out that it is very important to keep medical educators interested to improve the situation of MSK teaching. MSK conditions are usually overlooked though they represent $20 \%$ of both primary care and emergency-room visits of the most common health complications that require medical attention across the world. MSK disorders can lead to serious disabilities and significant burden and though its problems are expected to increase, there is a continuous neglect in musculoskeletal examination skills in clinical practice. To participate in solving this problem, the article accentuates the necessity of defining MSK competencies and of agreeing on standards of MSK exams and calls for an integrated teaching of MSK examination skills that uses interactive methodologies like patients' educators and peer-assisted learning. This article comprises four parts, an introduction, a description of the deficiencies in musculoskeletal examination skills, the causes of these deficiencies and finally some solutions of musculoskeletal examination skills deficiencies. We venture to bring attention to an overlooked but major analyses constituent that impacts patients' overall health.
\end{abstract}

Keywords: Examination; Musculoskeletal Examinations; Arthritis; Teaching

\section{Introduction}

This paper is a theoretical exploration of the problems of musculoskeletal (MSK) examination. We argue that although MSK symptoms are a most common health complications requiring medical attention and accounting to $20 \%$ of both primary care and emergency-room visits [1], MSK symptoms do not receive proper teaching attention. Hence, it is very important to keep medical educators interested to improve the situation of MSK teaching. The paper also questions the causes of these deficiencies and suggests some solutions to redeem this problem. In a recent study where a standardised MSK examination of the hand and wrist joints has been determined and validated, it was suggested that adopting such approach is hoped to improve clinicians' ability to diagnose arthritis at an early stage, particularly in areas where rheumatologists have limited access to ultrasound (US) [2]. This is one of the first studies to call attention to the persistence of the problem in Saudi Arabia specifically and to investigate causes of the problem and suggest educational solutions to remedy it for the benefit of the patients.

MSK conditions affect one in five adults [3]. In a

${ }^{*}$ Corresponding author. health survey, MSK disorders were ranked first in prevalence as the cause of chronic health problems, long term disabilities, and consultations with a health professional [1]. In Saudi Arabia, MSK disorders is the second major cause of outpatients visit in primary care centers and private clinics [3]. Low back pain is the most prevalent of musculoskeletal conditions; it affects nearly everyone at some point in time and about $4 \%-33 \%$ of the population at any given point [4]. MSK disorders are a very common cause of health problems. They result in limiting work in developed countries. Besides, up to $60 \%$ of people on early retirement or long term sick leave claim a MSK problem as the reason [5]. Furthermore, the impact of MSK conditions is predicted to increase dramatically in developing as well as developed countries with the aging of the population, changes in lifestyle resulting in obesity and lack of physical fitness, and the increase in road traffic accidents with the urbanization and motorization of the developing world [6]. This increasingly high impact of MSK conditions is recognized now by the United Nations, the World Health Organization, the World Bank, and many governments throughout the world through their support of the Bone and Joint Decade 2000-2011 initiative [7] that recognized MSK education 
as a national and global priority $[4,5,8]$ during the Bone and Joint Decade (2000-2010).

A number of different medical specialties are usually involved in treating patients with musculoskeletal complaints. This comprises general practitioners, family physicians, internists, orthopedic and surgeons. However, the various practitioners may work in teams with other health professional, but they often lack a multispecialty focus which results in treating the same patients in a segmented manner and from different inconsistent angles.

In the following part we are going to discuss the deficiencies in MSK examination and the causes of these deficiencies in order to arrive at a better understanding of this problem, its causes, and possible solutions to improve the MSK examination conditions.

\section{Deficiencies in Musculoskeletal Examination Skills}

Despite the impact of MSK disorders on health care, rheumatological diseases are often overlooked or inadequately assessed by doctors [9-12] and there is a continuous neglect observed in musculoskeletal examination skills in clinical practice. Thus problems of patients with complaints about bones and joints are often ignored and underestimated by doctors.

In a teaching hospital report, among 200 general medical inpatients only $5.5 \%$ of the signs and $14 \%$ of the symptoms of MSK disorder were recorded in the hospital examination notes. This compared poorly with recorded examination of other systems and regions; for example, cardiovascular symptoms were recorded in $100 \%$ of the cases; respiratory and abdominal symptoms were recorded in 99\%, the nervous system, skin and female breasts symptoms were recorded in $77 \%, 13 \%$, respectively [13]. In another report, only $40 \%$ of the patients admitted to the general medicine ward had the history of their MSK symptoms recorded and only $14.5 \%$ of these patients received comprehensive MSK examination [14]. Furthermore, $80 \%$ of symptomatic patients received either no treatment for their rheumatic disorder, or treatment that was regarded as suboptimal or inappropriate [14]. A third report shows even a higher percentage; 63\% of all patients admitted to the general medicine ward had MSK symptoms or its signs, but relevant MSK history was missed in $49 \%$ of the patients records and MSK signs were missed in $78 \%$; $42 \%$ of those with MSK conditions would have benefited from additional treatment [15]. A more recent report reviewed 150 patient notes in three different hospitals from the acute admission wards for medicine and surgery and the medical assessment unit. Factors considered included whether GALS screenings had taken place, documentation of MSK examina- tions and assessment of confidence of junior doctors in assessing MSK conditions. GALS screenings were performed for $4 \%$ of patients on the medical assessment unit, $7 \%$ of acute medical and $0 \%$ of acute surgical patients on admission. Examination of the MSK system yielded better results with $16 \%, 22 \%$ and $10 \%$ on each of the respective wards. Interviews with junior doctors found that $10 \%$ of the doctors routinely screened for MSK conditions though $87 \%$ felt confident in taking MSK histories [16]. Furthermore, Matzkin et al. (2005) indicate that the majority (79\%) of the study respondents including medical students, residents, and staff physicians failed the basic MSK cognitive examination [17]. This suggests that training in MSK medicine is inadequate in medical schools and in most residency training programs. Worldwide, undergraduate and postgraduate medical teaching of MSK disorders is brief currently and not directly relevant to the knowledge and skills commonly required for management of these conditions in an outpatient setting.

In undergraduate education, inadequate MSK education is reported. Medical students spend very few hours on the MSK system, both in basic science and in clinical training. It is quite common for students to leave medical school without being able to make a general assessment of the musculoskeletal system; on the other hand, it would be considered a total neglect if a medical graduate is incompetent at adequately assessing the heart or lungs. Harvard medical students report general dissatisfaction of their confidences in examining of MSK system comparing to their skill in examining pulmonary system [18] and they suggested more integration between pre-medical and clinical courses and more time to be devoted to MSK medicine.

The American Association of Medical Colleges, AAMC (2005) claims that most medical schools do not effectively educate future physicians on MSK medicine in spite of the increasing prevalence of MSK conditions across medical practice [19]. The obvious discrepancy between the magnitude of MSK conditions and physicians competences, which stemmed mostly from the educational deficiencies at the medical schools, continued across years [18-20]. Akesson and colleagues (2003) argue that MSK teaching at the undergraduate and graduate programs is not adequate and that the resulting competence does not reflect the impact of these conditions on individuals and society [10]. A comprehensive study reviewing the curricula of all Canadian medical schools indicated that directors of undergraduate MSK programs felt dissatisfied with the curricular time devoted to MSK education [21].

The same limitation subsists in postgraduate programs since the 1980s. Goldenberg et al. (1985) reported that the majority of directors of residency programs thought that many basic skills and techniques were not taught 
adequately and that the training of their rheumatology residents was not equal to that of residents in cardiology or gastroenterology [22]. General dissatisfactions of MSK training are reported in internal medicine residents and family practice. United State residents express their dissatisfaction of their competence in performing MSK examinations at various parts of the body and relate that to the inadequate or poor training [23]. Woolf et al. recommend a musculoskeletal undergraduate curriculum they developed to be applied in any country and culture [5]. They claim that the implementation of these recommendations is well suited to the trend to integrate courses both vertically and horizontally.

In a wide range study based on a national survey in Saudi Arabia using the Delphi technique, internal medicine knowledge and skills competencies including rheumatology were determined and prioritized [1]. In this study, MSK exam skills were considered part of core competencies that must be mastered by students at time of their graduation. A standardized approach to the clinical assessment of a musculoskeletal problem is suggested [24]. Such a standardized approach is to be conducted whether the patient is presented to primary care, rheumatology or orthopedics. The study also provides a benchmark for this competency that can be used as a teaching aid [24].

A major objection against this study is its standardized approach to the clinical assessment of a musculoskeletal problem [24]. It is considered that such standardization is an imposition in various medical disciplines. Therefore, it remains to be seen whether this kind of standardization would be widely accepted by different disciplines or not is debatable? The study does not address the important issue of the actual MSK examination techniques; how can a clinician perform a comprehensive and standard MSK examination of the hand and wrist joints for example? Feeling "over each joint line for tenderness and bony or synovial swelling” [24] may not be adequate enough in describing where to place the examiners hands? Where to press? What is considered a positive test? Unfortunately, there is no standard technique or approach to assess specifically any joint in the body, particularly if the concern was to identify an inflamed joint due to arthritis.

In response to this lack, in a recent study [2], definitions to an approach and techniques to examine the hand and wrist joints to diagnose arthritis as exemplified in Figures 1 and 2 was validated in comparison to ultrasound findings in patients presenting to rheumatology clinics. These figures illustrate three techniques that showed sensitivities ranges from $80 \%-70 \%$ to detect arthritis in comparison to ultrasound findings as gold standard.
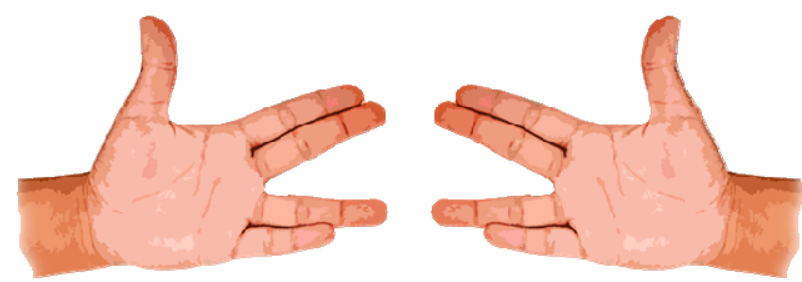

(a)

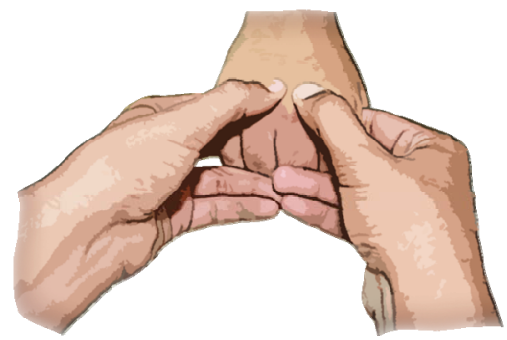

(b)

Figure 1. (a) MCP-scissor technique: first step, the examiner should make a scissor like shape with his/her fingers. (MCP: metacarpophalangeal joints); (b) MCP-scissor technique: second step, The examiner holds the patient hand from the sides at MCP level, flexing the MCPs to 90 degrees. The two free thumbs from both hands palpate the joint line for every MCP joint. One thumb is pressing firmly for a power causing whitening of the distal thumb nail while the other thumb is pushing intermittently in and out to assess for effusion, swelling and/or tenderness.

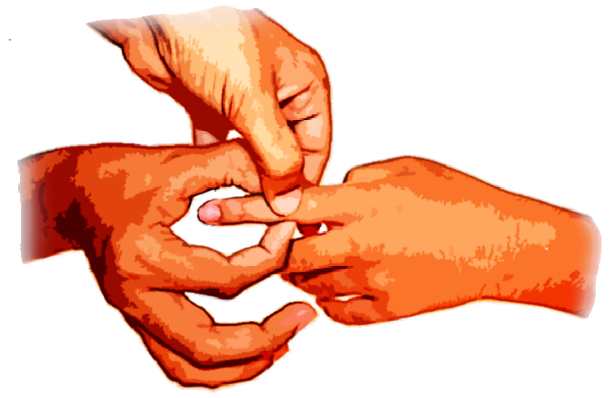

Figure 2. PIP-4 fingers technique: The examiner's thumb and index finger of one hand should hold each PIP from the side and press firmly until the whitening of distal fingers from low blood supply is clear. With the other thumb and index finger of the other hand, the examiner should hold the same PIP-joint from antro-posterior direction and push intermittently in and out to look for effusion, swelling and/or tenderness (PIP: proximal interphalangeal joints).

\section{Causes of Musculoskeletal Examination Skills Deficiencies}

Previous studies suggest many reasons related to MSK poor clinical skills and physical examinations in particular [3,10,18-20,24-28] such as:

- Vague training of MSK disorders in undergraduate programme.

- Examination of the MSK system is often regarded to 
be complex in comparison with other organ systems.

- Underestimation of the prevalence of MSK conditions and their impact on individuals and society.

- MSK disorders are not considered to be main competencies of medical graduates because they are not life threatening conditions.

- The lack of standardized approach to the clinical assessment of MSK problems, whether pertaining to primary care, rheumatology or orthopedics. Such standardize approach would present a competency benchmark.

- Lack of proper standard teaching in MSK disorders results in the low competence in MSK examination skills.

- Lack of summative evaluation of MSK examination skills contributes to low level of competency among medical graduates.

- The disparity in the approach to examination between rheumatologists and orthopaedic surgeons mostly lead to poor performances in MSK examinations.

- The lack of appropriate teaching and evaluation in MSK disorders; clinical teachers are not usually skilled in MSK examinations and thus bone and joint diseases are not screened.

\section{Solution of MSK Examination Deficiency}

We suggest that one of the basic steps in working through the obstacles of the deficiencies in MSK examination skills is to define competencies that should be mastered while dealing with MSK disorders and is to agree on what MSK skills should be mastered by medical students $[5,29]$. There is a comprehensive core recommendations developed for a musculoskeletal undergraduate curriculum to be applied in any country and culture [5]. It was claimed in this study that the implementation of these recommendations is therefore well suited to the trend to integrate courses both vertically and horizontally.

It is also important that experts in various specialties work more closely together and look for the commonality of approach when treating a patient as they often treat the same patients but from separate angles. Another solution would be an integrated MSK disease course for medical students, bringing together orthopedics, rheumatology, and physical medicine and rehabilitation has been found to be effective [29].

The method of teaching MSK examination skills should follow interactive approaches and hands-on teaching sessions where learners are involved in the teaching process. Patient educators can participate effectively in teaching MSK examination skills in different educational interventions [30-33]. Peer-assisted learning (a technique whereby students learn from and with each other) can be used to enhance MSK teaching for the un- dergraduate medical curricula [34-36].

\section{Standardization Problems Can Be Resolved in a Number of Ways}

A step towards standardizing MSK examination is to consider as an important step in every joint examination; a screening exam. It simply implies the examination of active range of motion of that joint where the patient should be able to demonstrate by himself a full range without pain or limitations. This is to assure maintenance of functionality, as diseased joints particularly due to inflammatory arthritis tend to lose the function early. GALS (Gait, Arms, Legs and Spine) is a MSK screening tool developed and validated as a rapid screening protocol/system for MSK system with the aim for a quick identification of significant abnormalities [37]. Various spectrums of health specialties could utilize this screening routine before specific examination of any joint can be conducted.

One of the essential steps in teaching MSK examination skills then would be to define an overall objective of examining this patient for a MSK problem. Examining a young patient for a knee joint pain following a sport injury should differ at least in the approach of the managing clinician in comparison to a young female complaining of small joints pains and swelling. In the former, it is important to assess joint stability as a major objective while in the latter, it is essential to examine for inflammatory arthritis. The screening exam for MSK abnormalities would be abnormal in both examples but then the objective of examining the first patient would be to evaluate for sport injuries, i.e. soft tissue problems while in the second patient would be evaluated for the presence of inflammatory arthritis. This is not to say that it is imperative to have a very limited MSK exam based on this objective, it is rather to be focused on the primary objective based on historical facts but at the same time, we emphasize that there is no substitute for a comprehensive approach to any joint examination.

The suggested approach should start with the screening examination of that joint then followed by inspection, palpation, range of motion testing (active and passive) and ends with special tests based on the clinician's objective on conducting this exam.

\section{Conclusion}

There is no doubt that MSK disorders are common. The prevalence of these disorders is expected to rise given the significant increase in our aging population. Despite the early reports about the neglect in basic MSK examination skills among clinicians, this process continues unfortunately. Several causes are described well in MSK literature and several attempts to overcome these obstacles 
have been designed and experimented with. Adjusting the way of teaching MSK examination skills by focusing on one general approach for conducting the exam then by focusing on a primary objective for examining individual patients should enable us to overcome many obstacles in evaluating MSK disorders. Furthermore, examination should be geared to patients' specific conditions. For example, a clinician attempting to evaluate a MSK problem should ask himself/herself: am I dealing with a sport injury (for example) or an inflammatory arthritis? The answer of this question should be based on careful historical points. The future direction of research should focus on defining and validating precise techniques of how to examine joints. However, in order to make any of this happen, a true interest and strong desire to overcome this observed deficiencies in MSK examination skills are required among medical educators.

\section{Acknowledgements}

The authors would like to thank Alzaidi Chair of Research in Rheumatic Diseases, Umm Alqura University for supporting this work and Dr. Khadeejah Bawazeer for reviewing the manuscript.

This work was funded and supported by Alzaidi Chair of Research in Rheumatic Diseases, umm Alqura University, Makkah, Saudi Arabia

\section{REFERENCES}

[1] H. Almoallim, "Determining and Prioritizing Competencies in the Undergraduate Internal Medicine Curriculum in Saudi Arabia,” Eastern Mediterranean Health Journal, Vol. 17, No. 8, 2011, pp. 656-662

[2] E. M. Badley, I. Rasooly and G. K. Webster, "Relative Importance of Musculoskeletal Disorders as a Cause of Chronic Health Problems, Disability, and Health Care Utilization: Findings from the 1990 Ontario Health Survey,” The Journal of Rheumatology, Vol. 21, No. 3, 1994, pp. 505-514.

[3] H. Almoallim, S. Attar, N. Jannoudi, N. Al-Nakshabandi, B. Eldeek, O. Fathaddien, et al., "Sensitivity of Standardised Musculoskeletal Examination of the Hand and Wrist Joints in Detecting Arthritis in Comparison to Ultrasound Findings in Patients Attending Rheumatology Clinics,” Clinical Rheumatology, 2012. doi:10.1007/s10067-012-2013-5

[4] MOH, "The Annual Health Report," 1430H-2009, Riyadh Ministry of Health, 2009.

[5] A. D. Woolf and B. Pfleger, "Burden of Major Musculoskeletal Conditions," Bulletin of the World Health Organization, Vol. 81, No. 9, 2003, pp. 646-656.

[6] A. D. Woolf, N. E. Walsh and K. Akesson, "Global Core Recommendations for a Musculoskeletal Undergraduate Curriculum,” Annals of the Rheumatic Diseases, Vol. 63, No. 5, 2004, pp. 517-524. doi:10.1136/ard.2003.016071
[7] A. D. Woolf and K. Akesson, "Understanding the Burden of Musculoskeletal Conditions. The Burden Is Huge and Not Reflected in National Health Priorities,” BMJ, Vol. 322, No. 7294, 2001, pp. 1079-1080. doi:10.1136/bmj.322.7294.1079

[8] A. D. Woolf, “The Bone and Joint Decade 2000-2010," Annals of the Rheumatic Diseases, Vol. 59, No. 2, 2000, pp. 81-82. doi:10.1136/ard.59.2.81

[9] J. Dequeker, J. J. Rasker and A. D. Woolf, "Educational Issues in Rheumatology, Bailliere's Best Practice \& Research,” Clinical Rheumatology, Vol. 14, No. 4, 2000, pp. 715-729.

[10] A. Jones, P. Maddison and M. Doherty, “Teaching Rheumatology to Medical Students: Current Practice and Future Aims," Journal of the Royal College of Physicians of London, Vol. 26, No. 1, 1992, pp. 41-43.

[11] K. Akesson, K. E. Dreinhofer and A. O. Woolf, "Improved Eduaction in Musculoskeletal Is Necessary for All Doctors,” Bulletin of Health Organization, Vol. 81, No. 9, 2003, pp. 677-682.

[12] H. Almoallim, E. Khojah, R. Allehebi and A. Noorwali, "Delayed Diagnosis of Systemic Lupus Erythematosus Due to Lack of Competency Skills in Musculoskeletal Examination,” Clinical Rheumatology, Vol. 26, No. 1, 2007, pp. 131-133.

[13] K. A. Beattie, R. Bobba, I. Bayoumi, D. Chan1, I. Schabort, P. Boulos, et al., "Validation of the GALS Musculoskeletal Screening Exam for Use in Primary Care: A Pilot Study," BMC Musculoskeletal Disorders, Vol. 9, 2008, pp. 115-122. doi:10.1186/1471-2474-9-115

[14] M. Doherty, J. Abawi and M. Pattrick, “Audit of Medical Inpatient Examination: A Cry from the Joint,” Journal of the Royal College of Physicians of London, Vol. 24, No. 2, 1990, pp. 115-118.

[15] M. J. Ahern, M. Soden, D. Schultz and M. Clark, "The Musculo-Skeletal Examination: A Neglected Clinical Skill," Australian and New Zealand Journal of Medicine. Vol. 21, No. 3, 1991, pp. 303-306. doi:10.1111/j.1445-5994.1991.tb04694.X

[16] M. S. Lillicrap, E. Byrne and C. A. Speed, "Musculoskeletal Assessment of General Medical In-PatientsJoints Still Crying Out for Attention,” Rheumatology, Vol. 42, No. 8, 2003, pp. 951-954. doi:10.1093/rheumatology/keg259

[17] D. Sirisena, H. Begum, M. Selvarajah and K. Chakravarty, "Musculoskeletal Examination-An Ignored Aspect. Why Are We Still Failing the Patients?" Clinical Rheumatology, Vol. 30, No. 3, 2010, pp. 403-407. doi:10.1007/s10067-010-1632-y

[18] E. Matzkin, E. L. Smith, D. Freccero and A. B. Richardson, "Adequacy of Education in Musculoskeletal Medicine,” The Journal of Bone and Joint Surgery American, Vol. 87, No. 2, 2005, pp. 310-314. doi:10.2106/JBJS.D.01779

[19] C. S. Day, A. C. YEh, O. Franko, M. Ramirez and E. Krupat, "Musculoskeletal Medicine: An Assessemnt of the Attituide and Knoweledge of Medical Studnets at Harverd Medical School,” Academic Medicine, Vol. 82, No. 5, 2007, pp. 452-457. 
doi:10.1097/ACM.0b013e31803ea860

[20] (AAMC) AoAMC, "Contemprory Issues in Medicine: Muscluskeletal Medicine Education,” Washington, 2005.

[21] M. L. Clark, C. R. Hutchison and J. M. Lockyer, "Musculoskeletal Education: A Curriculum Evaluation at One University,” BMC Medical Education, Vol. 10, No. 93, 2010. doi:10.1186/1472-6920-10-93

[22] S. Pinney and W. Regan, "Educating Medical Students about Musculoskeletal Problems: Are Community Needs Reflected in the Curricula of Canadian Medical Schools?” The Journal of Bone \& Joint Surgery, Vol. 83, No. 9, 2001, pp. 1317-1320.

[23] D. L. Goldenberg, J. K. Dehoratius, J. Mason, R. Meenan, S. G. Perlman and J. B. Winfield, "Rheumatology Training at Internal Medicine and Family Practice Residency Programs,” Arthritis \& Rheumatism, Vol. 28, No. 4, 1985, pp. 471-476. doi:10.1002/art.1780280420

[24] D. K. Clawson, D. W. Jackson and D. J. Ostergaard, “It's Past Time to Reform the Musculoskeletal Curriculum," Academic Medicine, Vol. 76, 2001, pp. 709-710. doi:10.1097/00001888-200107000-00012

[25] A. D. Woolf and K. Akesson, "Primer: History and Examination in the Assessment of Musculoskeletal Problems," Nature Clinical Practice Rheumatology, Vol. 4, No. 1, 2008, pp. 26-33. doi:10.1038/ncprheum0673

[26] J. Dequeker, G. Esselens and R. Westhovens, "Education Issues in Rheumatology. The Musculoskeletal Examination: A Neglected Skill,” Clinical Rheumatology, Vol. 26, 2007, pp. 5-7. doi:10.1007/s10067-006-0288-0

[27] E. Matzkin, E. Smith, D. Freccero and A. B. Richardson, "Adequacy of Education in Musculoskeletal Medicine," The Journal of Bone and Joint Surgery, Vol. 87, 2005, pp. 310-314.

[28] A. E. Thompson, "Improving Undergraduate Musculoskeletal Education: A Continuing Challenge,” The Journal of Rheumatology, Vol. 35, No. 12, 2008. doi:10.3899/jrheum.080972

[29] K. Saleh, R. Messner, S. Axtell, I. Harris and M. L. Mahowald, "Development and Evaluation of an Integrated Musculoskeletal Disease Course for Medical Students. The Journal of Bone and Joint Surgery American, Vol. 86,
No. A8, 2004, pp. 1653-1658.

[30] A. E. Oswald, J. Wiseman, M. J. Bell and L. Snell, "Musculoskeletal Examination Teaching by Patients Versus Physicians: How Are They Different? Neither Better nor Worse, but Complementary,” Medical Teacher, Vol. 33, No. 5, 2011, pp. e227-e235. doi:10.3109/0142159X.2011.557412

[31] N. Raj, L. J. Badcock, G. A. Brown, C. M. Deighton, S. C. O’Reilly, "Undergraduate Musculoskeletal Examination Teaching by Trained Patient Educators-A Comparison with Doctor-Led Teaching,” Rheumatology, Vol. 45, No. 11, 2006, pp. 1404-1408. doi:10.1093/rheumatology/kel126

[32] L. Schrieber, G. D. Hendry and D. Hunter, "Musculoskeletal Examination Teaching in Rheumatoid Arthritis Education: Trained Patient Educators Compared to Nonspecialist Doctors,” The Journal of Rheumatology, Vol. 27, No. 6, 2000, pp. 1531-1532.

[33] A. E. Oswald, M. J. Bell, J. Wiseman and L. Snell, "The Impact of Trained Patient Educators on Musculoskeletal Clinical Skills Attainment in Pre-Clerkship Medical Students,” BMC Medical Education, Vol. 11, No. 65, 2011. doi:10.1186/1472-6920-11-65

[34] K. Graham, J. M. Burke and M. Field, "Undergraduate Rheumatology: Can Peer-Assisted Learning by Medical Students Deliver Equivalent Training to That Provided by Specialist Staff?” Rheumatology, Vol. 47, No. 5, 2008, pp. 652-655. doi:10.1093/rheumatology/ken048

[35] J. Burke, S. Fayaz, K. Graham, R. Matthew and M. Field, "Peer-Assisted Learning in the Acquisition of Clinical Skills: A Supplementary Approach to Musculoskeletal System Training,” Medical Teacher, Vol. 29, No. 6, 2007, pp. 577-582. doi:10.1080/01421590701469867

[36] M. E. Perry, J. M. Burke, L. Friel and M. Field, "Can Training in Musculoskeletal Examination Skills Be Effectively Delivered by Undergraduate Students as Part of the Standard Curriculum?” Rheumatology, Vol. 49, No. 9, 2010, pp. 1756-1761. doi:10.1093/rheumatology/keq166

[37] M. Doherty, J. Dacer, P. Dieppe and M. Snaith, "The GALS Locomotor Screen," Annals of the Rheumatic Diseases, Vol. 51, 1992, pp. 1165-1169. doi:10.1136/ard.51.10.1165 